\title{
Démons et merveilles : les peuples monstrueux dans l'œuvre de Shakespeare
}

\section{Ladan Niayesh}

\section{OpenEdition}

\section{Journals}

\section{Édition électronique}

URL : http://journals.openedition.org/shakespeare/928

DOI : 10.4000/shakespeare.928

ISSN : 2271-6424

Éditeur

Société Française Shakespeare

\section{Édition imprimée}

Date de publication : 1 novembre 2002

Pagination : 149-164

Référence électronique

Ladan Niayesh, «Démons et merveilles : les peuples monstrueux dans l'œuvre de Shakespeare », Actes des congrès de la Société française Shakespeare [En ligne], 19 | 2002, mis en ligne le 01 novembre 2007, consulté le 01 mai 2019. URL : http://journals.openedition.org/shakespeare/928 ; DOI :

$10.4000 /$ shakespeare.928 


\section{S H A K E S P E A R E \\ \& L E M O Y E N - Â G E}

Société Française Shakespeare

Actes du Congrès de 2001

* * *

Textes réunis et présentés par

Patricia DORVAL

publiés sous la direction de Jean-Marie MAGUIN 


\section{DÉMONS ET MERVEILLES: LES PEUPLES MONSTRUEUX DANS L' CEUVRE DE S H K ES P EARE}

«Mandeville's Travels has a place in the history of travel as well as of literature», écrit Neil Rennie en guise d'introduction à une recherche qui s'intéresse moins aux Grands Voyages de découverte qu'à la manière dont ceux-ci étaient appréhendés à l'aune d'un héritage antique et médiéval par les hommes de la Renaissance ${ }^{1}$. Ce que N. Rennie affirme à propos des Voyages fictifs du pseudo-Jean de Mandeville peut aussi bien s'appliquer à une foule d'autres récits de voyages et d'écrits géographiques médiévaux plus ou moins fantaisistes qui, depuis le Roman d'Alexandre jusqu'à la «Lettre» factice du pseudo-Prêtre-Jean en passant par les différentes éditions, additions et interprétations de l'Histoire naturelle de.Pline l'Ancien jouissaient encore d'une grande popularité à la Renaissance et continuaient d'agir sur l'imaginaire d'une époque qui aurait dû les rejeter totalement au vu de ses découvertes géographiques.

Un rapide survol des titres de quelques-uns des plus célèbres Masques de cour sous les Tudors donne une idée de la survivance de ces légendes de contrées lointaines dans l'imaginaire anglais du XVIe siècle. Il y a là ce Mask of Prester John (1547-1548) qui, avec l'anonyme Tom of Lincoln (avant 1607) est l'une des deux pièces de la Renaissance anglaise à mettre en scène ce personnage légendaire de Prêtre-Roi chrétien des contrées sauvages de l'Asie ou de l'Éthiopie. Il y a encore The 
Cynocephali, masque de 1577 qui devait mettre en scène cette célèbre race plinienne d'hommes à tête de chien dont Mandeville avait attesté l'existence et dont Christophe Colomb avait longtemps cru avoir retrouvé la trace au Nouveau Monde. Citons également A Mask of Amazons (15511552, 1579), A Mask of Moors (1599) ou encore A Mask of Barbarians (1560), œuvres disparues dont les titres présagent d'une mise en scène de découvertes géographiques récentes aussi bien que de mythes et de légendes hérités de l'Antiquité et entretenus par maints auteurs médiévaux ${ }^{2}$.

Si, à l'exception notable des Amazones (présentes dans $A$ Midsummer Night's Dream, dans le masque de Timon of Athens et dans The Two Noble Kinsmen), le corpus des écrits dramatiques de William Shakespeare n'inclut à proprement parler aucun personnage issu des races pliniennes, les allusions à ces peuples et aux contrées lointaines qu'ils sont censés habiter se rencontrent dans la quasi-totalité des pièces de cet auteur. Nous y voyons les Anthropophages et les Blemmyes, ces hommes dont la tête se trouve au milieu de la poitrine et chez qui Othello aurait séjourné au cours de ses voyages : «The Anthropophagi, and men whose heads / Do grow beneath their shoulders» (Othello, I.3.143-4) ${ }^{3}$. Il y a encore les Cynocéphales, ces êtres mi-chiens, mi-singes, «dog-apes» ${ }^{4}$ auxquels les personnages de Jacques et d'Amiens se comparent dans As You Like It (II.5.22-6). Les Cyclopes, les Géants et les Pygmées sont respectivement mentionnés à deux, à onze et à quatre reprises dans les pièces de Shakespeare ${ }^{5}$. Quant au personnage de Benedick dans Much Ado About Nothing, il avoue préférer se rendre aux Antipodes pour prendre la mesure du pied de Prêtre-Jean plutôt qu'échanger trois mots avec cette harpie de Beatrice :

Will your grace command me to any service to the world's end? I will go on the slightest errand now to the Antipodes that you can devise to send me on. I will fetch you a tooth-picker now from the furthest inch of Asia, bring you the length of Prester John's foot, fetch you a hair of the Great Cham's beard, do you any embassage to the Pygmies, rather than hold three words' conference with this harpy. 
Rappelons qu'outre dans cet extrait, les Antipodes, ces régions diamétralement opposées à l'Europe sur la sphère terrestre et où toutes sortes de monstres sont censés vivre la tête en bas, sont mentionnés dans quatre pièces de Shakespeare.

Pourquoi de telles références à un savoir géographique dépassé apparaissent-elles si souvent sous la plume d'un auteur de la Renaissance ? D'où viennent ces légendes de peuples monstrueux et pourquoi les auteurs du XVIe et du XVIIe siècles éprouvent-ils encore le besoin de recourir à elles dans des écrits qui n'ont rien de géographique? Telles sont les axes de réflexion auxquels je propose de nous intéresser ici.

$$
* * *
$$

Dans son Shakespeare and the Geography of Difference, John Gillies insiste sur la dimension primordialement morale et symbolique de la géographie antique qu'il définit comme «une activité sans cesse renouvelée de différenciation» ${ }^{6}$. Pour les Grecs comme pour leurs héritiers latins, l'étude géographique apporte une preuve scientifique, ou pseudoscientifique, à la croyance en une différence essentielle et en une supériorité intrinsèque de leur espace (oikoumenê) par rapport à ces eschatia ou «terres de confins» où ils plaçaient toutes sortes de thomastâ, terme syncrétique par lequel ils désignaient le merveilleux aussi bien que ce «merveilleux à rebours» (l'expression est de Marie-Thérèse JonesDavies $^{7}$ ) qu'est le monstrueux ${ }^{8}$. De fait, pour les Anciens, la tératologie ou l'étude des monstres constituait une branche importante d'une géographie qui se voulait essentiellement humaine et qui, en partie du fait de ses visées idéologiques, accordait une place de choix à tout un fonds mythique ou légendaire.

Le savoir géographique grec s'appuyait dans une large mesure sur les récits de Ctésias (médecin grec à la cour des rois de Perses au Ve siècle avant Jésus-Christ et auteur d'une Indika dont ne subsistent que des fragments) et de Mégasthène (émissaire de Séleucos Ier à la cour du roi de l'Inde Chandragupta au début du IIIe siècle avant Jésus-Christ). Les informations plus ou moins fabulées de ces deux auteurs étaient corroborées par la prétendue «Lettre d'Alexandre le Grand à Aristote sur les merveilles de l'Inde» qui rapporte les campagnes d'Alexandre contre, puis aux côtés du roi de l'Inde Porus qui lui fait rencontrer maints peuples monstrueux ${ }^{9}$. Cette lettre est à l'origine du célèbre Roman d'Alexandre qui devait connaître un immense succès et de multiples versions et adaptations 
dans tout l'Occident au Moyen-Âge, voire jusqu'à la Renaissance. Il en existe au moins deux éditions en langue anglaise au XVIe siècle ${ }^{10}$. La plupart des races monstreuses prétendument rencontrées par Alexandre, comme les Cynocéphales, les Amazones, les Éthiopiens (aïth-ops, littéralement «visages brûlés», nullement situés en Afrique dans ce récit), les Géants, les femmes à barbe ou encore les Brahmans ou Gymnosophistes (les philosophes nus) se retrouvent aux livres VI et VII de l'Histoire naturelle de Pline l'Ancien, écrivain latin du début de l'ère chrétienne qui a tenté de compiler l'essentiel du savoir scientifique de son temps en matière d'histoire naturelle dans les trente-sept livres qui composent son ouvrage. Si Pline a retenu la quasi-totalité des races alexandrines, il a également contribué à en allonger considérablement la liste par l'adjonction d'autres peuples qu'il situe dans des régions autres que celles parcourues par Alexandre, dont l'Afrique ".

Si les légendes pliniennes répercutées par ses commentateurs ou ses héritiers plus ou moins directs tels que Solin (auteur de Polyhistor) ou Isidore de Séville (auteur d'Étymologies) devaient constituer le fondement du savoir géographique médiéval, elles n'en posaient pas moins un problème de taille à la doctrine chrétienne sur la création du monde et sur sa finalité et firent à ce titre couler beaucoup d'encre tout au long du Moyen-Âge. Saint Augustin est peut-être l'autorité théologique qui dans sa Cité de Dieu (XVI.8) s'est intéressé de plus près à ce qui semblait constituer un véritable défi à la conception monogénétique de la race humaine $^{12}$. Cette théorie, à laquelle le Père de l'Église adhère pleinement, veut que tous les hommes aient une même origine adamienne et puissent à ce titre prétendre pareillement au salut. Comment pouvait-on intégrer à cette vision du monde des monstres à l'existence attestée par des autorités alors irréfutables de l'Antiquité païenne? Dans ce schéma d'une création raisonnée et juste, quelle place pouvait-on accorder aux Panotii aux oreilles si immenses qu'ils pouvaient s'en servir comme d'une couverture, aux Sciopodes qui s'abritaient du soleil sous leur pied unique et démesurément grand, aux Astomi dépourvus de bouche et qui se sustentaient grâce au parfum des fruits ? L'existence de pareils monstres ne trouvait sa justification dans le dessein général de la création que si l'on faisait correspondre une explication rationnelle ou une leçon morale à chacune de leurs caractéristiques monstrueuses. Et c'est à cette tâche que s'emploient, à l'instar d'Augustin et d'Isidore, maintes autorités théologiques et/ou géographiques de l'Occident médiéval. 
Deux hypothèses alternatives étaient communément avancées par les auteurs médiévaux pour rendre compte de l'existence des peuples monstrueux. La première était qu'il s'agissait de créatures réprouvées par Dieu, de descendants de quelque grande figure de transgresseur biblique, comme Caïn ou comme Cham, le fils maudit de Noé. Ainsi, leur monstruosité physique était-elle censée refléter les péchés de leurs ancêtres et une dégénérescence morale. La seconde explication consistait à voir dans ces peuples des incarnations de vices et de vertus divers. Cette explication conférait aux monstres une fonction didactique dans l'œuvre de création. Un exemple de la première théorie serait constitué par ces mappemondes médiévales en forme de $\mathrm{T}$ enchâssé dans un cercle et que John Block Friedman appelle des cartes tripartites ou noachides car elles sont supposées refléter la division du monde entre les trois fils de Noé. Dans ces cartes, les races maudites sont séparées des autres par une frontière aquatique qui rappelle symboliquement le Déluge et qui les maintient loin des espaces et des espèces civilisés ${ }^{13}$. Un exemple de la seconde théorie explicative quant à la monstruosité physique de certains peuples serait un chapitre de la Gesta Romanorum, un célèbre recueil d'histoires pseudo-antiques compilées par un moine franciscain anglais anonyme du milieu du XIVe siècle. Dans ce texte qui se place largement dans la lignée interprétative des Bestiaires, l'auteur fait correspondre une caractéristique morale, tantôt positive et tantôt négative, à chacun des traits physiques distinctifs des peuples qu'il énumère. Ainsi les Blemmyes auraient-ils leur tête au milieu de la poitrine comme une marque de leur humilité. Les grandes oreilles des Panotii les rendraient plus réceptifs à la Parole de Dieu, alors que les Cyclopes ne suivraient que l'œil unique de la raison. À l'opposé, les hommes dépourvus de nez seraient également dépourvus de discernement, tandis que la beauté physique des Pygmées prouverait qu'une entreprise bien commencée peut malgré tout tourner court ${ }^{14}$. Le point commun entre tous ces peuples, qu'ils soient présentés comme admirables ou comme condamnables, c'est qu'ils véhiculent un message moral à l'usage de l'homme chrétien et européen.

C'est peut-être là que réside la cause du succès immense dont jouissent ces peuples monstrueux dans l'art figuratif du Moyen-Âge. Dans son ouvrage La Nature et les prodiges, Jean Céard reproduit à titre d'exemples plusieurs représentations de la Pentecôte dans l'art médiéval, dont celle qui figure sur le tympan du grand portail de la basilique de la Madeleine à Vézelay (XIIe siècle). Autour d'un compartiment central où l'on voit le Christ envoyer sur les apôtres l'Esprit-Saint, on aperçoit une 
série de petites scènes figurant plusieurs races monstrueuses, comme les Cynocéphales, les Panotii et les Pygmées ${ }^{15}$. Pendant inversé du récit de la tour de Babel et de la confusion des langues dans l'Ancien Testament, cette représentation de la Pentecôte participe de la doctrine augustinienne du salut accessible à tous les fils d'Adam, tout autant qu'elle accrédite l'hypothèse de la monstruosité de certains de ces fils.

Cet engouement pour le monstrueux devait connaître un essor encore plus important sous l'effet des Croisades, des pélerinages, des missions papales et des échanges commerciaux avec l'Orient. Aux récits authentiques de marchands comme Marco Polo, d'ambassadeurs comme Guillaume de Rubroek ou de missionnaires tel qu'Odoric de Pardenone ${ }^{16}$ s'ajoutent des sources fantaisistes encore plus sensationnelles, comme la prétendue «lettre» du Prêtre-Jean ou les Voyages de Sir John Mandeville. Le premier de ces documents, qui apparait dans un contexte de propagande en faveur des Croisades, est une missive adressée à l'empereur byzantin Manuel Ier Comnène et à l'empereur germanique Frédéric Ier Barberousse en 1163. L'auteur, probablement un faussaire nestorien, se présente comme le monarque chrétien d'une riche et puissante contrée de l'Inde peuplée de races monstrueuses, mais toutes converties au christianisme, qui propose son concours aux croisés dans leur lutte contre les Infidèles. Maintes fois reproduite au Moyen-Âge et au-delà, cette lettre et la légende du PrêtreJean acquièrent bientôt une renommée et un statut comparables à ceux du Roman d'Alexandre dans l'imaginaire occidental ${ }^{17}$. Le second document se présente comme le récit des voyages d'un pseudo-chevalier de Saint Albans, Sir John Mandeville, qui aurait parcouru trois continents entre 1322 et 1356 et rencontré des peuples tels que les Amazones, les Cynocéphales, les Blemmyes et les Pygmées au cours de ses voyages en Égypte, en Éthiopie, en Chine, aux Antipodes chez le Prêtre-Jean et jusqu'aux sources du Tigre et de l'Euphrate, tout près des portes du Paradis. Expérience extrême, l'exploration des limites du monde apparaît aussi et surtout comme une exploration des limites de l'humain pour cet auteur de l'un des récits de voyages les plus célèbres de l'Occident médiéval.

L'immense popularité de ce texte, dont attestent ses quelque deux cent cinquante manuscrits médiévaux écrits en diverses langues européennes ${ }^{18}$, en fait l'une des autorités géographiques les plus incontournables à l'aube de l'ère des Grands Voyages de découverte. Ce texte et les croyances syncrétiques héritées du savoir et de l'idéologie antiques et médiévaux qu'il véhicủe font partie intégrante de l'imaginaire 
européen de la fin du Moyen-Âge et de celui des découvreurs du Nouveau Monde à la Renaissance. À en croire les biographes contemporains, les Voyages de Mandeville auraient trouvé une place dans les bagages de Christophe Colomb, découvreur de l'Amérique, tout autant que redécouvreur de maintes légendes mandevilliennes dans ce cadre nouveau. L'anecdote de l'invention du terme «cannibale» par Christophe Colomb est trop connue pour qu'on s'y attarde longuement ici. Je rappelle simplement que, croyant avoir atteint l'Asie, Colomb a longtemps cru que les terribles indigènes «caribes» ou «canibes» des Antilles n'étaient autres que les sujets cynocéphales et anthropophages du Grand Khan, puisqu'une étymologie fantaisiste lui faisait reconnaître les racines «can» (khan) et «canis» (chien en latin) dans le nom «canibe» ${ }^{19}$.

$\mathrm{Au}$ nombre des célèbres possesseurs d'un volume des Voyages de Mandeville, signalons encore Martin Frobisher, le grand navigateur élisabéthain qui emporta ce récit fantaisiste comme document géographique authentique à une date aussi tardive que 1576 dans son voyage à la recherche du légendaire passage du Nord-Ouest vers l'Asie. Les races mandevilliennes paraissaient encore suffisamment crédibles en 1589 pour que Richard Hakluyt fasse figurer les Voyages de Mandeville dans la première édition des Principal Navigations, Voyages and Discoveries of the English Nation. Hakluyt retire ce récit fabuleux de la seconde édition corrigée de 1598-1599, mais quasiment à la même époque, Walter Raleigh croit, à la suite de Francisco de Orellana, avoir localisé plusieurs races mandevilliennes en Guyane, dont les Amazones et les Blemmyes :

Such a nation was written of by Mandeville, whose reports were held for fables many years, and yet since the Indies were discovered, we find his relations true of such things as heretofore were held incredible. ${ }^{20}$

Naturellement, ni Colomb, ni Raleigh plus d'un siècle après lui ne parviendront jamais à rencontrer personnellement ces peuples monstrueux dont ils entendent seulement parler par les indigènes. Certes, la crédulité affichée par Raleigh représente une tendance minoritaire au tournant du XVIIIe siècle et c'est peut-être ce même passage que Shakespeare parodie quelques années plus tard dans une réplique de Gonzalo dans The Tempest : 
When we were boys,

Who would believe [...] there were such men

Whose heads stood in their breasts? Which now we find

Each putter-out of five for one will bring us

Good warrant of.

(III.3.43-9)

Néanmoins, l'exemple de Raleigh et la date tardive de sa citation montrent à quel point le regard porté sur le nouveau continent par les grands voyageurs n'était pas innocent, mais sélectif et à l'affût d'éléments susceptibles de confirmer l'existence de vieilles croyances médiévales dans un décor nouveau. Et quand les voyageurs ne parviennent pas à trouver la confirmation tant souhaitée, comme ce fut si souvent le cas, ils se contentent d'assigner une nouvelle localisation géographique à leurs données légendaires, les situant dans l'au-delà immédiat des territoires qu'ils viennent d'explorer. Tout comme les cynocéphales anthropophages de Colomb ou les Amazones et les Blemmyes de Raleigh, le monstre se trouve toujours une île, une montagne ou un village plus loin.

$* * *$

La littérature dramatique de la Renaissance anglaise reproduit ce même schéma d'attente sans cesse cultivée, déçue, puis relancée dans ses multiples allusions aux peuples monstrueux. Poursuivis avec la même assiduité et la même fascination que celles de Desdemona «dévorant»les récits d'Othello sur les Anthropophages et les Blemmyes ${ }^{21}$, les peuples monstrueux n'apparaissent jamais directement sur scène ailleurs que dans les Masques ${ }^{22}$. Et tout comme ces masques monstrueux cachent en réalité les premiers personnages du royaume, la monstruosité dans une pièce telle qu'Othello surgit pour finir, non dans un ailleurs fabuleux, mais au cœur de la cité et sous le vernis de civilité du plus vénitien des Maures.

C'est ainsi que dans l'œuvre de Shakespeare, nous trouvons rarement les monstres là où nous nous attendons le plus à les trouver. Hippolyta, la terrible reine des Amazones tueuses d'hommes apparaît dans deux pièces de Shakespeare, A Midsummer Night's Dream et The Two Noble Kinsmen, fruit d'une collaboration avec John Fletcher. Mais dans les deux cas, elle déçoit l'amateur du sensationnel et du monstrueux en se présentant comme un «fantasme masculin d'une féminité sauvage apprivoisée» ainsi que l'appelle Jeanne Addison Roberts ${ }^{23}$, autrement dit en étant sur le point de 
renoncer à sa monstruosité et d'accepter sa condition de femme en s'unissant à Thésée.

De la même manière, The Tempest, la seule pièce de Shakespeare à mettre en scène un Nouveau Monde, à défaut $d u$ Nouveau Monde, se montre étonnamment pauvre en matière d'indigènes monstrueux. Ce n'est pas faute d'avoir fortement envisagé l'hypothèse de leur présence. «Do you put tricks upon's with savages and men of Ind, ha ?» s'écrie Stefano à la vue du monstre quadrupède formé par Trinculo et Caliban réfugiés sous une même gabardine (II.2.57-9). Mais l'une comme l'autre partie de ce monstre bicéphale vont bientôt s'avérer être des enfants de l'Ancien, plutôt que du Nouveau Monde, puisque même Caliban, en apparence le seul indigène de l'île de Prospero, est en réalité le fils de la sorcière d'Alger Sycorax. Le cri d'étonnement de Miranda «O brave new world / That has such people in't !» (V.1.186-7) n'est nullement poussé à la vue de quelque merveille issue d'un continent nouveau, mais au contact des habitants de l'Ancien Monde, seuls objets de découverte sur l'île du magicien. En réalité; tout se passe dans cette pièce comme si les mirabilia véritables ne se trouvaient pas dans un ailleurs légendaire, mais bien chez nous.

Ce trait semble constituer une constante du traitement des allusions aux peuples monstrueux par Shakespeare. Nous ne les trouvons jamais aussi fréquemment que dans les pièces les plus «anglaises» de cet auteur, celles dont l'intrigue se passe indubitablement sur le sol anglais et met en scène des compatriotes de Shakespeare. Citons à cet effet l'exemple extrême des pièces faisant intervenir Falstaff, le plus anglais des bouffons shakespeariens, qui est volontiers comparé à un Anthropophage dans The Merry Wives of Windsor: «He'll speak like an Anthropophaginian unto thee», dit l'Hôtelier à son propos (IV.5.8). Ailleurs, dans 2 Henry IV, Falstaff s'adresse en ces termes antinomiques à son petit page : «Sirrah, you giant, what says the doctor to my water ?» (I.2.1-2). Ailleurs encore, le blafard médecin français Doctor Caius qui s'apprête à se battre en duel contre le Gallois Sir Hugh Evans est encouragé par l'Hôtelier qui l'appelle, admiratif, «my Ethiopian» (II.3.25). Le point commun entre toutes ces références est qu'elles servent à souligner, non ce que sont les personnages, mais ce qu'ils ne sont certainement pas. À chaque fois, c'est le décalage par rapport au modèle monstrueux, et par là même fascinant, qui contribue à souligner la petitesse et le ridicule des personnages. Tout comme les monstres d'Isidore de Séville ou de la Gesta Romanorum, ces noms exotiques ne représentent aucun intérêt en eux-mêmes, mais trouvent 
tout leur sens du fait d'une application à un personnage d'Européen. L'Autre ici n'est jamais qu'un miroir anamorphotique nous révélant notre vérité profonde.

Ce phénomène, dont le personnage de Falstaff et les pièces où il apparaît fournissent une illustration comique, n'est pas absent non plus des tragédies. Ainsi peut-on remarquer que les références aux peuples monstrueux sont particulièrement fréquentes dans les scènes de folie et de délire où se révèlent d'autres facettes, plus sauvages et plus primitives, des personnages. Ainsi le roi Lear dans la pièce qui porte son nom et la fille du geôlier dans The Two Noble Kinsmen en appellent-ils, l'un aux Géants et l'autre aux Pygmées. «There's my gauntlet. I'll prove it on a giant», s'écrie Lear dans sa rage impuissante (IV.5.90), tandis que la fille du geôlier, déçue par les hommes, aspire à un sort meilleur chez les Pygmées : «then would I make / A carrack of a cockle-shell, and sail / By east and north-east to the King of Pygmies» (III.4.13-5).

Mais la métaphore filée la plus remarquable en termes de référence aux races légendaires dans l'œuvre de Shakespeare se trouve peut-être dans une autre scène de King Lear, celle où le vieux roi se trouve sur la lande balayée par la pluie et le vent en compagnie de son bouffon, de Kent, et surtout d'Edgar déguisé en mendiant fou. La genèse du déguisement de «Tom o'Bedlam» adopté par le personnage d'Edgar et les nombreux intertextes qu'impliquent les scènes du mendiant fou ont fait l'objet de gloses multiples et conséquentes dans de nombreuses éditions de King Lear. Il y a pourtant un intertexte qui semble avoir échappé à ces nombreux éditeurs. À l'acte III, scène 4 , c'est-à-dire la scène où «Poor Tom», le mendiant fou et nu est présenté au roi déchu, celui-ci l'appelle spontanément "philosopher», une appellation qu'il utilise à trois reprises dans cette scène : «First let me talk with this philosopher. / What is the cause of the thunder ?» (III.4.144-5), «Noble philosopher, your company» (162) et «With him ! / I will keep still with my philosopher» (165). En fait, tout se passe comme si la nudité du personnage suffisait à faire de lui un philosophe. On pense aux philosophes cyniques, naturellement, et tout particulièrement à Diogène qui demeurait nu dans son tonneau. Mais l'identification à Diogène est rendue impossible par une autre précision fournie par Lear : «I'll talk a word with this same learnèd Theban» (147). Diogène est natif de Sinope en Asie Mineure, et non de Thèbes. Le seul philosophe nu que l'Antiquité associe à Thèbes est Crates, contemporain de Diogène. Est-ce de lui qu'il s'agit, comme le pense R.A. Foakes, éditeur de la pièce pour la collection «Arden» en 1997? ${ }^{24}$ Son 
prédécesseur Kenneth Muir, qui avait édité la pièce pour la même collection en 1972, relevait une occurrence similaire dans une pièce de Jonson, Pan's Anniversary: "Then comes my learned Theban, the Tinker, I told you of» (vii.532) Soulignant une parenté possible entre les deux emplois, il se disait pourtant incapable d'en expliquer le sens : «This may be an echo of this scene; but it looks as though both Jonson and Shakespeare were using an expression, the meaning of which has been lost» ${ }^{25}$.

Pour moi, ces deux passages s'éclairent à la lecture d'un chapitre des Voyages de Mandeville dans l'édition de 1582 qui est chronologiquement la plus proche de la composition de King Lear. Il s'agit de la description d'une île légendaire de l'océan Indien, celle où réside une célèbre race alexandrine et plinienne : «some men call this Ile the Ile of Bragamen, and some call it the land of faith, and through it runneth a great riuer that men call Thebe...» ${ }^{26}$. Ces «Bragamen» ou Brahmans, également connus sous le nom de Gymnosophistes (philosophes nus), font essentiellemnt parler d'eux dans les ouvrages de géographie ou les récits de voyages médiévaux en rapport avec l'épopée d'Alexandre. En effet, l'un des passages les plus connus du Roman d'Alexandre correspond à la rencontre du grand conquérant avec cette race pacifique dont il entend envahir le territoire. Mais les gymnosophistes qui vivent dans le dénuement et la renonciation la plus totale vis-à-vis du monde, et qui passent leurs journées dans la contemplation méditative du soleil, surprennent l'élève d'Aristote en répondant à toutes ses questions philosophiques. Ils donnent ainsi une leçon de sagesse et d'humilité au conquérant qui renonce à envahir leur territoire et rebrousse chemin.

Présentés tantôt comme les témoins d'un Âge d'Or et tantôt comme des chrétiens avant l'heure, les gymnosophistes font partie des races incontournables dans la plupart des écrits moralisateurs du Moyen-Âge ayant recours au topos des races légendaires ${ }^{27}$. Ainsi, utilisant l'intertexte de la rencontre du monarque et des philosophes nus, la rencontre de Lear et du mendiant participe de la veine didactique dans le traitement des allusions aux peuples légendaires. Le gymnosophiste de Lear n'existe que sous la forme d'un déguiisement adopté par un Européen pour donner une leçon d'humilité à d'autres Européens, qu'ils soient des personnages ou des spectateurs de la pièce.

Un même intertexte peut avoir présidé à l'élaboration de la scène de la rencontre de Timon d'Athènes avec Alcibiades et son armée à l'acte IV, scène 3 de Timon of Athens, une pièce légèrement antérieure à King Lear. 
Nous y voyons Timon, le plus grec, le plus athénien des gymnosophistes, vivant à demi nu et dans le dénuement le plus total dans la forêt, où il trouve par hasard une cache d'or. Survient le glorieux général Alcibiades, accompagné de son armée et sur le point de lancer une attaque vengeresse contre Athènes. L'humilité et la renonciation de Timon à toute idée de vengeance donnent une leçon morale, quand bien même excentrique et excessive à Alcibiades qui par la suite saura se montrer magnanime après la conquête de la ville.

L'intervention de l'or dans cet épisode évoque peut-être également une autre rencontre d'Alexandre, cette fois avec les Amazones qui, outre les gymnosophistes, semblent avoir été les seules à avoir donné une cuisante leçon au conquérant macédonien et ainsi arrêté son avancée. En effet, à Alexandre qui leur demande du pain, elles tendent une miche en or pour lui prouver la vanité des richesses. De la même manière, dans Timon of Athens, à Alcibiades qui offre de l'or («Here is some gold for thee», IV.3.101), Timon répond : «Keep it. I cannot eat it» (101). Il les renvoie, lui et sa suite, en leur offrant encore plus d'or, tout comme les Amazones du Roman d'Alexandre décourageaient le conquérant par leur offrande de pain en or. C'est peut-être cette référence indirecte aux Amazones dans cette pièce qui a motivé l'inclusion d'un Masque des Amazones à l'acte I, scène 2, Masque que le philosophe cynique Apemantus ponctue de ses commentaires acerbes en guise de memento mori :

I should fear those that dance before me now Would one day stamp upon me. 'T'as been done. Men shut their doors against a setting sun.

$$
* * *
$$

Dans son traitement du topos des races légendaires, Shakespeare est loin de représenter une tendance minoritaire au sein des dramaturges anglais de la Renaissance. En dépit d'allusions nombreuses, rares sont les auteurs dramatiques de cette période à mettre directement en scène des peuples monstrueux issus de l'imaginaire antique et médiéval ou des légendes remaniées associées au Nouveau Monde. Et quand ils représentent effectivement de telles merveilles sur scène, comme le font John Fletcher et Philip Massinger des Amazones et des Anthropophages qui apparaissent dans leur pièce The Sea Voyage (1622) ou Massinger seul 
avec les Indiens cannibales de sa comédie The City Madam (1632), c'est presque toujours pour faire intervenir un coup de théâtre ou une scène de reconnaissance qui finit par montrer que ceux qu'on a d'abord pris pour des monstres sont en fait des Européens déguisés ou ensauvagés dans un contexte hostile.

Du latin monere (montrer, avertir), le monstre est celui qu'on montre, mais aussi celui qui montre, voire qui démontre quelque chose aux autres. Suivant la double étymologie de son nom, le monstre des écrits géographiques de l'Antiquité et du Moyen-Âge, aussi bien que de la littérature, notamment dramatique, de la Renaissance remplit essentiellement une fonction d'avertissement moral en mettant en évidence la sauvagerie et la monstruosité qui se cachent sous. le vernis de civilité de son double européen.

Ladan NIAYESH Université de Paris VII / C.E.R.R.A.

\section{NOTES}

${ }^{1}$ Neil Rennie, Far-Fetched Facts: The Literature of Travel and the Idea of the South Seas, Oxford, Clarendon Press, 1995, p. 16.

${ }^{2}$ Les dates et les titres de ces divertissements de cour, tous disparus aujourd'hui, sont ceux qu'indiquent Alfred Harbage et Samuel Schoenbaum dans Annals of English Drama: 975-1700, Londres, Methuen, 1974. Quant à Tom of Lincoln, pièce nouvellement redécouverte sous la forme d'un manuscrit de scribe, il a été réédité par John Pitcher pour la collection des «Malone Society Reprints», Oxford, Oxford University Press, 1992.

${ }^{3}$ Toutes les citations tirées des œuvres de Shakespeare renvoient à l'édition de Stanley Wells et de Gary Taylor, The Complete Oxford Shakespeare, Oxford, Oxford University Press, 1987.

${ }^{4}$ L'Oxford English Dictionary définit ce terme comme «a dog-faced baboon, Cynocephalus». 
${ }^{5}$ Pour ces informations et les suivantes, voir les entrées correspondantes dans Marvin Spevack, A Complete and Systematic Concordance to the Works of Shakespeare, Hildesheim, Georg Olms, 1970.

${ }^{6}$ John Gillies, Shakespeare and the Geography of Difference, Cambridge, Cambridge University Press, 1994 : «a ceaselessly renewed activity of differentiation» (p. 6).

${ }^{7}$ Marie-Thérèse Jones-Davies, «Le Monstre, expression de l'insécurité dans la littérature et les spectacles de la Renaissance anglaise», p. 26-41, in MarieThérèse Jones-Davies (éd.), Monstres et prodiges au temps de la Renaissance, Paris, Jean Touzot, 1980, p. 26.

8 Voir à ce sujet Gillies, p. 8, ainsi que François Hartog, Le Miroir d'Hérodote : Essai sur la représéntation de l'autre, Paris, Gallimard, 1980, en particulier p. 31 et suivantes.

${ }^{9}$ Sur la genèse des légendes de peuples monstrueux dans l'imaginaire grec, voir John Block Friedman, The Monstrous Races in Medieval Art and Thought, Cambridge, Harvard University Press, 1981, chapitre II : «A Measure of Man» (p. 26-36).

${ }^{10}$ A Romance of King Alexander, Londres, R. Faques, 1525, STC 321 ; The Book of Alexander the Great, Édimbourg, A. Arbuthnet, c. 1580, STC 321.5.

${ }^{11}$ L'ouvrage de Pline était accesssible à la Renaissance anglaise à travers la splendide édition in-folio de la traduction de Philemon Holland, The History of the World, commonly called, the Naturall Historie of C. Plinius Secundus, Londres, Adam Islip, 1601, STC 20029.

${ }^{12}$ Une traduction anglaise de ce texte était disponible au début du XVIIe siècle : Of the citie of God: with the learned comments of J. L. Vives, tr. J. H[ealey], Londres, G. Eld, 1610, STC 916. Livre XVI, chapitre 8 : Sigs. Ddd2vDdd3v.

13 Voir à ce sujet l'étude que Friedman fait des célèbres cartes d'Hereford (1290) et d'Ebstorf (c. 1240) : Monstrous Races, chapitre III : «At the Round Earth's Imagined Corners» (p. 37-58).

${ }^{14}$ Voir pour détails Gesta Romanorum, tr. Charles Swan, révis. Wynnard Hopper, Londres, George Bell and Sons, 1899 : «Tale CLXXV : Of the World's Wonders» (p. 337-40). Il convient de préciser que la Gesta Romanorum connut plusieurs traductions et éditions partielles à l'époque élisabéthaine, mais qu'aucune n'inclut ce conte.

15 Jean Céard, La Nature et les prodiges : L'insolite au XVle siècle, en France, Genève, Droz, 1977, p. 49.

${ }^{16}$ Marco Polo, marchand vénitien, aurait pris en 1271 la route de la soie et aurait atteint la Chine pour rencontrer l'empereur Kubilay Khan en 1275. Celui-ci lui ayant confié diverses missions, Polo aurait parcouru le pays pendant seize ans avant de rentrer à Venise (1295), où il rédige son Livre des merveilles du monde ou Il Milione. Guillaume de Rubroek, franciscain flamand, fut envoyé par Saint Louis 
en mission auprès du grand khan, qu'il rencontra en 1254. Il a laissé une relation de voyage fort complète. Odoric de Pardenone, franciscain italien, a effectué un voyage de plusieurs années en Mongolie, en Chine et en Inde à la fin du XIIIe et au début du XIVe siècles. Il a rédigé une Descriptio terrarum ou Itinerarum.

${ }^{17}$ Une version du XIIIe siècle de cette lettre se trouve à la Bibliothèque Nationale de France (MS. 9634). Elle est publiée par A. Jubinal en annexe de son édition des Euvres complètes de Rutebeuf, Paris, P. Daffis, 1875 : tome III, p. 355-75.

${ }^{18}$ Voir à ce sujet l'Introduction de Christiane Deluz à sa traduction de Mandeville, Voyage autour de la terre, Paris, Les Belles Lettres, 1993, p. xv.

${ }^{19}$ Voir à titre d'exemple un passage du Journal de Bord du voyage de 14921493 retranscrit par Bartolomé de Las Casas, le premier biographe de Colomb : «Il dit que tous ces gens rencontrés jusqu'à aujourd'hui ont une immense crainte des caniba ou canima. [...] Ils disaient que les caniba n'avaient qu'un seul œil et une face de chien. L'Amiral pensait qu'ils mentaient et croyait que ceux qui les capturaient étaient de la seigneurie du Grand Khan» ( $L a$ Découverte de l'Amérique, vol. I, tr. Soledad Estorach et Michel Lequenne, Paris, François Maspéro, 1981 : Jeudi 26 novembre 1492 : p. 117.)

${ }^{20}$ Sir Walter Raleigh, The Discovery of the Large, Rich and Beautiful Empire of Guiana, 1596, éd. Gerald Hammond, Selected Writings, Manchester, Carcanet, 1984, p. 111.

${ }^{21}$ «She'd come again, and with a greedy ear / Devour up my discourse» (Othello, I.3.148-9).

${ }^{22}$ Signalons, outre les Masques disparus mentionnés au début de cette étude, The Mask of the Middle Temple and Lincoln's Inn (1613) de George Chapman qui figure des princes virginiens, des esclaves indiens et maures, ainsi que des babouins habillés en hommes, ou encore Tempe Restored (1631) d'Aurelian Townshend où les Indiens rejoignent les «barbares», les lions, les singes et les chiens pour un antimasque figurant les désordres de l'âme.

${ }^{23}$ «... the male fantasy of the wild female tamed...» (Jeanne Addison Roberts, The Shakespearean Wild: Geography, Genus, and Gender, Lincoln et Londres, University of Nebraska Press, 1991, p. 130).

${ }^{24}$ R.A. Foakes (éd.), King Lear, «The Arden Shakespeare», Londres, Thomas Nelson, 1997 : note de bas de page correspondant à III.4.150 dans cette édition.

${ }^{25}$ Kenneth Muir (éd.), King Lear, «The Arden Shakespeare», Londres, Methuen, 1972 : note de bas de page correspondant à III.4.154 dans cette édition.

${ }^{26}$ The Voyages and Trauailes of Sir John Mandeville, tr. anonyme, Londres, Thomas Este, 1582, STC 645 : Sig. S4v.

${ }^{27}$ Voir à cet effet Friedman, p. 167 et 171. Pour un exemple de présentation moralisatrice de cette race et d'autres races légendaires, voir Thomas de 
Cantimpré, De Naturis Rerum, livre III : «De Monstruosis Hominibus» (circa 1290). Références fournies par Friedman, p. 126. 